\title{
Elimination of GInKAmtB affects serine biosynthesis and improves growth and stress tolerance of Escherichia coli under nutrient-rich conditions
}

Romina Frare ${ }^{1}$, Margarita Stritzler ${ }^{1}$, Cecilia Pascuan ${ }^{1}$, Karen Liebrenz ${ }^{1}$, Luisa GalindoSotomonte ${ }^{1}$, Gabriela Soto ${ }^{1}$, Pablo Iván Nikel ${ }^{2}$, Nicolás Ayub ${ }^{1^{*}}$

1 Instituto de Agrobiotecnología y Biología Molecular (IABIMO), Instituto de Genética (IGEAF), INTA-CONICET, Buenos Aires, Argentina.

${ }^{2}$ The Novo Nordisk Foundation Center for Biosustainability, Technical University of Denmark, Kgs. Lyngby, Denmark.

* Corresponding author:

https://www.scopus.com/authid/detail.uri?authorld=6507695334

www.researchgate.net/profile/Nicolas_Ayub

https://orcid.org/0000-0001-5012-240X

Address: De los Reseros S/N, Castelar C25(1712), Buenos Aires, Argentina

E-mails: nicoayub@gmail.com

Telephone: 54-11-44500805-136

Fax: 54-11-44500805

Keywords: evolutionary innovation; ammonium uptake; lag phase; growth rate; oxidative stress.

\section{Abstract}

Nitrogen is a most important nutrient resource for Escherichia coli and other bacteria that harbor the glnKamtB operon, a high-affinity ammonium uptake system highly interconnected with cellular metabolism. Although this system confers an advantage to bacteria when growing under nitrogen-limiting conditions, little is known about the impact of these genes on microbial fitness under nutrient-rich conditions. Here, the genetically tractable $E$. coli BW25113 strain and its gInKamtB-null mutant (JW0441) were used to analyze the impact of GInK-AmtB on growth rates and oxidative stress tolerance. Strain JW0441 showed a shorter initial lag phase, higher growth rate, higher citrate synthase activity, higher oxidative stress tolerance and lower expression of serA than strain BW25113 under nutrient-rich conditions, suggesting a fitness cost to increase metabolic plasticity associated with serine metabolism. The overexpression of serA in strain JW0441 resulted in a decreased growth rate and stress tolerance in nutrient-rich conditions similar to that of strain BW25113, suggesting that the 
negative influence on bacterial fitness imposed by GInK-AmtB can be traced to the control of serine biosynthesis. Finally, we discuss the potential applications of glnKamtB mutants in bioproduction processes.

\section{Introduction}

Natural environments normally exhibit an extremely high microbial diversity, nutrient limitations, and a variety of abiotic stress conditions (Ayub et al., 2004; Ayub et al., 2009; Pascuan et al., 2015; Soto et al., 2012; Stritzler et al., 2018). Accordingly, the growth rate, nutrient uptake and adaptation to abiotic stress are crucial parameters that reflect the fitness of microbes in their natural habitat. Nitrogen is an elemental component of almost all macromolecules in a microbial cell (Reitzer, 2003), including nucleic acids, proteins and cell wall compounds. Consequently, prokaryotes possess complex control mechanisms to provide an optimal nitrogen amount for cellular metabolism and to survive in nitrogen-limiting conditions (Kustu et al., 1984; van Heeswijk et al., 2013).

Ammonium (found in an aqueous equilibrium between the protonated and the neutral species, i.e. $\mathrm{NH}_{4}{ }^{+}$and $\mathrm{NH}_{3}$ ) is the optimal nitrogen source for prokaryotes that contain the glnKamtB operon (e.g. Escherichia coli) and other ammonium uptake systems. The gInKamtB operon encodes a sensitive sensory system that regulates ammonium influx in response to external alterations in ammonium availability (Coutts et al., 2002). AmtB is an ammonium transporter whose activity is negatively regulated by the reversible formation of a complex with GInK (Javelle et al., 2004), a small cytosolic signal transduction protein (PII protein) that orchestrates the activities of the main regulators of carbon and nitrogen metabolism (Burkovski, 2003; Gosztolai et al., 2017; Kim et al., 2012; van Heeswijk et al., 2013; Vo et al., 2013). E. coli contains yet another PII protein, termed GInB, with a different function than the GInK protein from which it derives (Reitzer, 2003; Thomas et al., 2000). This is not surprising, largely due to the long-term maintenance of paralogs needed to foster the occurrence of neofunctionalization and/or subfunctionalization processes (Conant and Wolfe, 2008).

Based on the genetic linkage and presence of the glnK and amtB genes in strains belonging to the Bacteria and Archaea domains, it has been proposed that these genes constitute an ancestral ammonium-sensing system of prokaryotes (Javelle and Merrick, 2005; Thomas et al., 2000). However, since horizontal gene transfer is a critical mechanism of microbial evolution (Fox et al., 2014; Pascuan et al., 2015; Stritzler et al., 2018), the presence of this system in the Bacteria and Archaea domains does not necessarily imply that its emergence occurred in the common ancestor of prokaryotes. In addition, although several studies have demonstrated the importance of the ammonium-sensing system in the 
adaptation of microbes to changes in nutrient availability, including starvation and nutrientshock conditions (Gosztolai et al., 2017; Kidd and Wingreen, 2010; Kustu et al., 1984), little is known about the fitness cost of the occurrence of this nitrogen regulatory system.

Thus, the aim of this study was to integrate phylogenetic and functional analyses to characterize the emergence of the $g / n K$ and $a m t B$ genes in prokaryotes and to understand the occurrence and conservation of these genes together with their possible impact of on microbial fitness, focusing on growth rate and oxidative stress tolerance as key parameters.

\section{Material and Methods}

\section{Bacterial strains and genomic analyses}

Escherichia coli BW25113, a fast-growth model strain without auxotrophies, and its $\Delta$ InKamtB derivative (strain JW0441, CGSC\#: 8602), obtained from the Coli Genetic Stock Center (Baba et al., 2006), were used in this study. The integrity of the glnKamtBoperon in strain BW25113 and the knockout of this operon by the insertion of a kanamycin cassette in strain JW0441 were verified by sequencing and analyzing their genomes by means of Illumina Hiseq1500 technology and Geneious as indicated previously (Brambilla et al., 2019).

\section{Plasmid construction, bacterial transformation and gene expression}

Plasmids pECgInKamtB and pNOD26 were constructed for homologous and heterologous complementation of ammonium uptake deficiency in strain JW0441, respectively. Firstly, a PCR was carried out to amplify a fragment containing the glnKamt operon of strain BW25113 by using primers OpFW (5'-ATCTGCAGGCCAGCGTGCGTGAAGAGGAAT-3') and OpRV (5'-ACCGTTCAGGAAGgGGTGATGCGTAAT-3'). The primers flanked a 1,955-bp fragment including the entire glnKamt operon and its intergenic regions. This amplification fragment was cloned into vector pGEM- $t$ Easy (Promega \#A1360) and its identity was verified by sequencing (Cuyeu et al., 2013). The resulting plasmid was termed pECgInKamtB. Secondly, plasmid pNOD26, carrying the ammonium channel NOD26 gene from Glycine max (Frare et al., 2018), was constructed by introducing amplified and Xbal-digested fragments of the fulllength NOD26 cDNA into the E. coli-expressing vector pSEVA4413 (Silva-Rocha et al., 2013). The primers used were $26 a F W$ (5'-CCTCTAGAATGGCTGATTATTCAGCAGG-3') and 26aRV (5'CCICTAGATTATTTGGAGGCAGCACGgC-3'), which contain Xbal sites. The absence of mutations in the NOD26 gene was verified by sequencing. In addition, the serA gene from strain BW25113 was amplified using primers 5'-CCTCTAGAATGGCAAAGGTATCGCT-3' and 5'CCAAGCTTTTAGTACAGCAGACGGG-3' and cloned in the expression vector pSEVA2513 (Silva- 
Rocha et al., 2013) by using Xbal and Hindlll restriction enzymes. The resulting plasmid was named pSEVA-serA. Plasmids pECgInKamtB, pNOD26 and pSEVAserA were introduced by transformation into competent cells of strain JW0441. Ampicillin-resistant (JW0441pECgInKamtB), streptomycin-resistant (JW0441-pNOD26) and kanamycin-resistant (JW0441-pSEVAserA) transformants were maintained on LB agar plus ampicillin (100 $\mu \mathrm{g} / \mathrm{mL})$, streptomycin $(100 \mu \mathrm{gm} / \mathrm{L})$ and kanamycin $(50 \mu \mathrm{g} / \mathrm{mL})$. qRT-PCR experiments were performed according to (Setten et al., 2013). 16S rRNA gene was chosen as the internal control gene.

\section{Bacterial growth under nitrogen-limiting conditions}

To test growth under nitrogen-limiting conditions, overnight cultures grown in lysogeny broth (LB) medium were washed twice in physiological solution and serially diluted

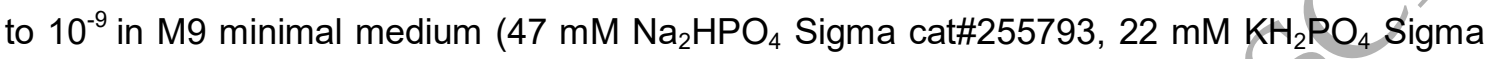
cat\#P9791, $20 \mathrm{mM} \mathrm{NH}_{4} \mathrm{Cl}$ Sigma cat\#213330, $8.5 \mathrm{mM} \mathrm{NaCl}$ Ciardelli cat\#750325, $1.9 \mathrm{mM}$ $\mathrm{MgSO}_{4}$ Sigma cat\#746452, $0.09 \mathrm{mM} \mathrm{CaCl}_{2}$ ICN cat\#195088, 27 mM glucose Biopack cat\#9638.08, $\mathrm{pH}=7.4)$. Then, aliquots $(0.1 \mathrm{~mL}$ each) were plated in solid M9 medium (M9 medium supplemented with $1.5 \%(\mathrm{w} / \mathrm{v})$ Agar Sigma cat\#A7921) varying both the concentration of nitrogen (from $0.01 \mathrm{mM}$ to $20 \mathrm{mM} \mathrm{NH}_{4} \mathrm{Cl}$ ) and the $\mathrm{pH}$ values (5.5 or 7.4) in the last medium and incubated at $37^{\circ} \mathrm{C}$ for 60 days. As a control of the presence of bacterial cells in each sample, aliquots $(0.1 \mathrm{~mL}$ each) were also plated onto LB agar. Experiments showing $<10$ or $>200$ colony-forming units (CFU) in this control were discarded.

\section{Growth rate, citrate synthase activity and oxidative stress assays}

Cultures were performed in 125 -mL Erlenmeyer flasks containing $25 \mathrm{~mL}$ of LB medium, incubated at $37^{\circ} \mathrm{C}$ with shaking (250 rpm). Overnight cultures grown at $37^{\circ} \mathrm{C}$ were used to inoculate fresh media at an initial optical density (OD $580 \mathrm{~nm}$ ) of 0.05 . Bacterial growth, doubling time and citrate synthase activity of strains were assessed under this nutrient-rich environment. Growth was monitored by measuring optical density for 135 min, and doubling time was evaluated in exponentially growing cells, where citrate synthase activity was measured by the CSA kit (Sigma-Aldrich CS0720). Tolerance to $\mathrm{H}_{2} \mathrm{O}_{2}$ was measured as previously described (Ayub et al., 2004), with slight modifications. Sterile Whatman No. 1 filter disks $(5 \mathrm{~mm})$ impregnated with $5 \mu \mathrm{L}$ of $10 \%(\mathrm{w} / \mathrm{w}) \mathrm{H}_{2} \mathrm{O}_{2}$ were placed on top of bacteria-seeded plates. Inhibition zones were measured after incubation at $37^{\circ} \mathrm{C}$ for 24 


\section{Bioinformatic protein and phylogenetic analyses}

GInK (NP_414984) and AmtB (NP_414985) protein sequences of Escherichia coli were used as query to search against the genome database of NCBI (https://www.ncbi.nlm.nih.gov/) by using BLASTP software. Protein identities were calculated using MatGAT (Campanella et al., 2003). The glnK and amtB genes are normally, but not always, co-localized in the genome. Bioinformatic studies were restricted to homologous proteins ( $>25 \%$ amino acid identity) as previously explained (Perez Di Giorgio et al., 2014). For each microbial species, we selected the proteins with highest amino acid identity with GInK and AmtB from E. coli. The accession numbers of these proteins are shown in Fig. 1. Individual phylogenetic trees of GInK and AmtB proteins were constructed using the Neighbor-Joining (NJ) method with genetic distances computed using the p-distance model and bootstrap analysis of 500 resamples and root on midpoint, using the MEGA software (Stecher et al., 2020). A consensus tree of GInK and AmtB proteins using individual trees were constructed using the SplitsTree4 software (Huson and Bryant, 2006) by setting standard parameters as previously (Brambilla et al., 2020).

\section{Results and Discussion}

To investigate the origins of the glnK and amtB genes in prokaryotes, we analyzed the presence and the evolutionary relationships of these proteins from sequenced species belonging to the Bacteria and Archaea domains. Putative homologous proteins ( $>25 \%$ amino acid identity), which contain conserved motifs of the GnIK and AmtB proteins, are ubiquitous in Bacteria (thirty-two phyla) but almost absent (one phylum, Euryarchaeota) in Archaea (Fig. 1). Naturally, despite their evolutionary equivalence, some of these putative homologous proteins probably display functional divergence. For example, GlnB proteins show a large functional divergence with respect to their homologous-derived GInK proteins (Thomas et al., 2000). The phylogenetic analysis showed that the GInK and AmtB proteins from the Bacteria and Archaea domains are not divergent lineages and that these proteins from archaeal strains cluster with different bacterial phyla (Fig. 1). The most parsimonious explanation for this result is the emergence of the glnK and amtB genes in a common ancestor of Bacteria and their occasional transfer to Archaea. Interestingly, we observed complete congruence (i.e. same topology) between the GInK-AmtB tree and the organismal evolution at the phylum level within Bacteria (Fig. 1). This fact suggests that the glnK and amtB genes from strains belonging to different bacterial phyla were inherited by vertical transfer. Since the number of possible evolutionary trees grows exponentially with the number of taxa studied, the probability that the congruent pattern observed in Bacteria occurs by chance is practically 
null (Perez Di Giorgio et al., 2014). Considering that our evolutionary analysis supported that the presence of $g I n K$ and $a m t B$ is an ancestral feature of the Bacteria domain, the retention, long-term persistence, and conservation of these genes suggest a robust contribution of this high-affinity ammonium uptake system to the bacterial fitness.

To explore the potential impacts of the gInKamtB operon on metabolic plasticity, the growth of the wild-type strain Escherichia coli BW25113 and its derived glnKamtB mutant strain JW0441 was analyzed in solid M9 minimal medium using nitrogen-limiting conditions (Table 1). These experimental conditions included variable ammonium $\left(\mathrm{NH}_{4}{ }^{+}+\mathrm{NH}_{3}\right)$ concentrations (from 0.01 to $20 \mathrm{mM}$ ) at two $\mathrm{pH}$ values $\left(5.5\right.$ and 7.4). $\mathrm{NH}_{4}^{+}$needs an ammonium channel to enter the cell, while $\mathrm{NH}_{3}$ crosses the cell membrane by unmediated diffusion (van Heeswijk et al., 2013). At pH 5.5, ammonium ( $\mathrm{pK}_{\mathrm{a}}=9.25$ ) is mostly protonated $\left(\mathrm{NH}_{3}\right.$ only accounts for $0.02 \%$ of the total ammonium), and then, nitrogen limitation is stronger for cells lacking ammonium channels (Frare et al., 2018; Vo et al., 2013). As expected, strains BW25113 and JW0441 showed similar vigorous growth at high ammonium concentrations $(\geq 0.5 \mathrm{mM})$, independently of the $\mathrm{pH}$ of the medium, and strain JW0441 exhibited slower growth than strain BW25113 at low ammonium concentrations $(\leq 0.25 \mathrm{mM})$ (Table 1). Specifically, at low ammonium ( $\geq 0.1 \mathrm{mM}$ ) and low $\mathrm{pH}(5.5)$, strain JW0441 was unable to grow (Table 1). In this extreme condition, the behaviour of the complemented strain JW0441/pECgInKamtB (homologous complementation) was similar to that of the wild type strain BW25113 (Table 2), further supporting a critical role of the ammonium channel AmtB under nitrogen-limiting conditions. Similar results have been previously described in a Bacillus subtilis background, where the presence of the $n r g A B$ operon, encoding homologs of AmtB and GInK (Thomas et al., 2000), is necessary for ammonium uptake under nitrogenlimiting or low pH conditions (Detsch and Stülke, 2003). Complementation of the mutant strain JW0441 with plasmid pNOD26 carrying the ammonium channel NOD26 from Glycine max (heterologous complementations) also restored the ability of the mutant cells to grow under these strict nitrogen-limiting conditions (Table 2). Taken together, these results verify the relevance of an ammoniumm channel to enable a high metabolism plasticity. It is interesting to note that the strain JW0441/pNOD26 showed higher percent of viable cells than the strains BW25113 and JW0441/pECgInKamtB under extreme nitrogen deficiency at low $\mathrm{pH}$ condition (Table 2). This is probably due the plant passive channel NOD26, which facilitates the diffusion of ammonium in favor of the concentration gradient in symbiotic nodules, was selected in a particularly acid (e.g. $\mathrm{pH}=5$ ) microenvironments (anoxic root cells) (Frare et al., 2018).

In addition to its contribution to the uptake of ammonium under nitrogen-limiting conditions, the occurrence of the glnK and $a m t B$ in bacterial cells also implies an integration of the modulation of carbon and nitrogen metabolisms (Kidd and Wingreen, 2010; Kustu et 
al., 1984). This co-occurrence could potentially affect other important parameters reflecting the fitness and competitiveness of bacteria such as the maximal growth rate. The last point is particularly important because the intrinsic constraints of bacterial maximal growth rate remains unclear (Zhu and Dai, 2018). In this context, we studied the growth and oxidative stress tolerance of strains either containing or lacking the gInKamtB operon in rich liquid LB medium, where growth rate is usually unrestricted. The mutant strain JW0441 showed a shorter initial lag and a higher duplication time than its parental strain BW25113 (Fig. 2). The results observed for the complemented strain JW0441/pECgInKamtB were similar to those described for the wild type strain (Fig. 2), supporting the notion that the glnKamtB operon plays a role on growth phenotypes.

The lag phase is a stress period that prepares bacteria for cell division, and its extension is positively correlated with the control of cellular damage (Bertrand, 2019). Specifically, oxidative stress is a distinctive feature of the lag phase (Bradley et al., 2007; Cuny et al., 2007) and genetic modifications that increase oxidative stress tolerance of bacteria notably reduce the duration of lag periods (Ayub et al., 2009). In this context, we decided to explore the impact of the lack of the glnKamtB operon on the resistance to oxidative stress. Strain JW0441 exhibited a higher oxidative stress tolerance than strain BW25113 (Fig. 3a). Complementation of the mutant strain JW0441 with the pECglnKamtB plasmid suppressed the stress-tolerant phenotype of strain JW0441 (Fig. 3a), a fact that verifies the negative impact of this ammonium uptake system in abiotic stress tolerance and that provides a mechanism to explain the lag phase reduction in strain JW0441.

Citrate synthase (CS) catalyzes the first reaction of the tricarboxylic acid cycle (TCA), playing a critical roles in central carbon and energy metabolism. Developmental roles of CS have been described in divergent bacterial lineages (Ireton et al., 1995; Viollier et al., 2001; Vornhagen et al., 2019; Zalis et al., 2019) and some lower eukaryotes (Kim et al., 1986; Ruprich-Robert et al., 2002). Thus, we decided to evaluate the effect of the lack of the glnKamtB operon on the activity of CS as a marker of metabolic robustness. Strain JW0441 showed increased CS activity compared to strains BW25113 and JW0441/pECgInKamtB in exponentially growing cells (Fig. 3b), suggesting that the loss of GInK and AmtB enhances activities through the TCA cycle.

Previous studies have shown that the glnK mutation leads to an increase in the expression of the nitrogen assimilation control (NAC) protein under both nitrogen-limiting and nitrogen-excess conditions (Blauwkamp and Ninfa, 2002b) and that NAC mediates the repression of the expression of serA under nitrogen-rich conditions (Blauwkamp and Ninfa, 2002a) (Fig. 4a). The serA gene encodes D-3-phosphoglycerate dehydrogenase, an enzyme that catalyzes the first and rate-limiting step of the L-serine biosynthesis pathway from intermediates of glycolysis (Zhang et al., 2017; Zhao and Winkler, 1996). Then, L-serine 
synthesis drains off metabolites from glycolysis. Although around $15 \%$ of the glycolytic flux is directed into the L-serine synthetic pathway in E. coli, only $6 \%$ of this carbon flow is used for protein synthesis (McKitrick and Pizer, 1980; Pizer and Potochny, 1964). This is because Lserine is a central cellular intermediate, which serves as a precursor for synthesis of important metabolites such as other amino acids (e.g. glycine, cysteine and tryptophan), purines, pyrimidines, phospholipids and C1 units (Grant, 2018; Peters-Wendisch et al., 2002). In addition to its metabolic functions, L-serine can act as a signalling molecule that stimulates the expression of stress response genes and can be used as a precursor in the biosynthesis of gene products involved in adaptation to abiotic stress conditions (Kitamoto et al., 2020; Matthews and Neidhardt, 1989). Thus, L-serine catabolism can improve bacterial fitness under the pressure of different environmental stressors (Fig. 4a). Considering this background and our results, we propose that GInK can moderate the growth rate and stress tolerance under nitrogen-rich conditions through the indirect induction of serA expression. In accordance with this hypothesis, the mutant strain JW0441 exhibited lower expression of the serA gene than its parental strain BW25113 and complemented strain JW0441/pECgInKamtB (Fig. 4a). Transformation of the JW0441 mutant with plasmid pSEVA2513-SerA, containing the serA gene under the control of a constitutive promoter, reversed the rapid growth (Fig. 4b) and stress-tolerant (Fig. 4c) phenotype of this strain.

Regardless of the evolutionary mechanism of the maintenance of the glnK and amtB genes in bacteria, which is probably related to recurrent exposures to nitrogen-limiting conditions in natural environments, fitness costs associated with the presence of these genes in optimal growth conditions could be a serious obstacle to their conservation in bacterial populations. Thus, the reduced gInKamtB expression under nutrient-rich conditions (Atkinson et al., 2002) could be interpreted as a mechanism to mitigate the cost of the maintenance of these genes under favourable environmental conditions. On other hand, under controlled conditions, the presence of the glnK and amtB genes could negatively affect the efficiency of recombinant bacteria as cell factories for the biosynthesis of natural and artificial products. In fact, the growth rate and general stress resistance are critical factors, for example, in the efficiency of $E$. coli in fermentation processes (Liu et al., 2015; Yang et al., 2020). Specifically, oxidative stress resistance in E. coli plays a critical role in the production of biofuels (Chen et al., 2018; Koppolu and Vasigala, 2016). In this line, by monitoring biomass increase on the basis of OD $580 \mathrm{~nm}$ values, higher cell densities were observed in JW0441 $(9.1 \pm 0.2, n=3)$ compared to BW25113 $(O D=7.1 \pm 0.3, n=3)$ after $24 \mathrm{~h}$ of culture in LB medium (i.e. in the stationary phase). Thus, the experimental design of metabolically engineered $E$. coli for the production of different compounds can include the knockout of the glnK and $a m t B$ genes to maximize its efficiency. Further studies involving other bacterial species and gInKamtB mutant strains are necessary to determine whether the alteration in 
growth and stress tolerance via knockout of these genes, as that described in this work, is a general mechanism to produce improved microbial cell factories.

\section{Conclusions}

The benefits of possessing a high-affinity ammonium uptake system highly interconnected with the carbon and nitrogen metabolisms for coordination of microbial growth under variable nutrient availability have been known since the identification of the $g \operatorname{lnK}$ and amtB genes in bacteria. However, the origin and fitness cost of this evolutionary innovation have not yet been analyzed. In this study, we provide evidence of the emergence and conservation of these genes in the Bacteria domain, and confirmed their importance under certain nitrogen-limiting conditions. Interestingly, we showed that the presence of this ammonium uptake system implies an intrinsic constraint on the growth rate and stress tolerance of bacteria. This occurrence marks an unique example of a nutrient uptake system limiting the potential maximal growth rate of microbes.

Acknowledgements. This work was supported by Grant PICT-2017-0674 provided to NA.

\section{References}

Atkinson, M.R., Blauwkamp, T.A., Bondarenko, V., Studitsky, V., Ninfa, A.J., (2002) Activation of the $\mathrm{g} \ln \mathrm{A}, \mathrm{g} \operatorname{\mathrm {nK}}$, and nac promoters as Escherichia coli undergoes the transition from nitrogen excess growth to nitrogen starvation. J Bacteriol 184, 5358-5363.

Ayub, N.D., Pettinari, M.J., Ruiz, J.A., Lopez, N.I., (2004) A polyhydroxybutyrate-producing Pseudomonas sp. isolated from Antarctic environments with high stress resistance. Curr Microbiol 49, 170-174.

Ayub, N.D., Tribelli, P.M., Lopez, N.I., (2009) Polyhydroxyalkanoates are essential for maintenance of redox state in the Antarctic bacterium Pseudomonas sp. 14-3 during low temperature adaptation. Extremophiles 13, 59-66.

Baba, T., Ara, T., Hasegawa, M., Takai, Y., Okumura, Y., Baba, M., Datsenko, K.A., Tomita, M., Wanner, B.L., Mori, H., (2006) Construction of Escherichia coli K-12 in-frame, single-gene knockout mutants: the Keio collection. Mol Syst Biol 2, 20060008.

Bertrand, R.L., (2019) Lag Phase Is a Dynamic, Organized, Adaptive, and Evolvable Period That Prepares Bacteria for Cell Division. J Bacteriol 201.

Blauwkamp, T.A., Ninfa, A.J., (2002a) Nac-mediated repression of the serA promoter of Escherichia coli. Mol Microbiol 45, 351-363.

Blauwkamp, T.A., Ninfa, A.J., (2002b) Physiological role of the GlnK signal transduction protein of Escherichia coli: survival of nitrogen starvation. Mol Microbiol 46, 203-214.

Bradley, M.D., Beach, M.B., de Koning, A.P.J., Pratt, T.S., Osuna, R., (2007) Effects of Fis on Escherichia coli gene expression during different growth stages. Microbiology 153, 2922-2940.

Brambilla, S., Frare, R., Stritzler, M., Soto, G., Berini, C., Jozefkowicz, C., Ayub, N., (2020) Synthetic multi-antibiotic resistant plasmids in plant-associated bacteria from agricultural soils. J Glob Antimicrob Resist. 
Brambilla, S., Soto, G., Odorizzi, A., Arolfo, V., McCormick, W., Primo, E., Giordano, W., Jozefkowicz, C., Ayub, N., (2019) Spontaneous Mutations in the Nitrate Reductase Gene napC Drive the Emergence of Eco-friendly Low-N2O-Emitting Alfalfa Rhizobia in Regions with Different Climates. Microb Ecol.

Burkovski, A., (2003) Ammonium assimilation and nitrogen control inCorynebacterium glutamicumand its relatives: an example for new regulatory mechanisms in actinomycetes. FEMS Microbiology Reviews 27, 617-628.

Campanella, J.J., Bitincka, L., Smalley, J., (2003) MatGAT: an application that generates similarity/identity matrices using protein or DNA sequences. BMC Bioinformatics 4, 29.

Chen, C.T., Chen, F.Y., Bogorad, I.W., Wu, T.Y., Zhang, R., Lee, A.S., Liao, J.C., (2018) Synthetic methanol auxotrophy of Escherichia coli for methanol-dependent growth and production. Metab Eng 49, 257-266.

Conant, G.C., Wolfe, K.H., (2008) Turning a hobby into a job: how duplicated genes find new functions. Nature reviews. Genetics 9, 938-950.

Coutts, G., Thomas, G., Blakey, D., Merrick, M., (2002) Membrane sequestration of the signal transduction protein GlnK by the ammonium transporter AmtB. EMBO J 21, 536-545.

Cuny, C., Lesbats, M., Dukan, S., (2007) Induction of a global stress response during the first step of Escherichia coli plate growth. Appl Environ Microbiol 73, 885-889.

Cuyeu, R., Rosso, B., Pagano, E., Soto, G., Fox, R., Ayub, N.D., (2013) Genetic diversity in a world germplasm collection of tall fescue. Genet Mol Biol 36, 237-242.

Detsch, C., Stülke, J., (2003) Ammonium utilization in Bacillus subtilis: transport and regulatory functions of NrgA and NrgB. Microbiology (Reading, England) 149, 3289-3297

Fox, A.R., Soto, G., Mozzicafreddo, M., Garcia, A.N., Cuccioloni, M., Angeletti, M., Salerno, J.C., Ayub, N.D., (2014) Understanding the function of bacterial and eukaryotic thiolases II by integrating evolutionary and functional approaches. Gene 533, 5-10.

Frare, R., Ayub, N., Alleva, K., Soto, G., (2018) The Ammonium Channel NOD26 is the Evolutionary Innovation that Drives the Emergence, Consolidation, and Dissemination of Nitrogen-Fixing Symbiosis in Angiosperms. J Mol Evol 86, 554-565.

Gosztolai, A., Schumacher, J., Behrends, V., Bundy, J.G., Heydenreich, F., Bennett, M.H., Buck, M., Barahona, M., (2017) GInK Facilitates the Dynamic Regulation of Bacterial Nitrogen Assimilation. Biophys J 112, 2219-2230.

Grant, G.A., (2018) D-3-Phosphoglycerate Dehydrogenase. Frontiers in Molecular Biosciences 5. Huson, D.H., Bryant, D., (2006) Application of phylogenetic networks in evolutionary studies. Mol Biol Evol 23, 254-267.

Ireton, K., Jin, S., Grossman, A.D., Sonenshein, A.L., (1995) Krebs cycle function is required for activation of the SpoOA transcription factor in Bacillus subtilis. Proc Natl Acad Sci U S A 92, 28452849.

Javelle, A., Merrick, M., (2005) Complex formation between AmtB and GInK: an ancestral role in prokaryotic nitrogen control. Biochem Soc Trans 33, 170-172.

Javelle, A., Severi, E., Thornton, J., Merrick, M., (2004) Ammonium sensing in Escherichia coli. Role of the ammonium transporter AmtB and AmtB-GlnK complex formation. J Biol Chem 279, 8530-8538.

Kidd, P.B., Wingreen, N.S. (2010) Modeling the role of covalent enzyme modification in Escherichia coli nitrogen metabolism. Phys Biol 7, 016006.

Kim, K.S., Rosenkrantz, M.S., Guarente, L., (1986) Saccharomyces cerevisiae contains two functional citrate synthase genes. Mol Cell Biol 6, 1936-1942.

Kim, M., Zhang, Z., Okano, H., Yan, D., Groisman, A., Hwa, T., (2012) Need-based activation of ammonium uptake in Escherichia coli. Mol Syst Biol 8, 616.

Kitamoto, S., Alteri, C.J., Rodrigues, M., Nagao-Kitamoto, H., Sugihara, K., Himpsl, S.D., Bazzi, M., Miyoshi, M., Nishioka, T., Hayashi, A., Morhardt, T.L., Kuffa, P., Grasberger, H., El-Zaatari, M., Bishu, S., Ishii, C., Hirayama, A., Eaton, K.A., Dogan, B., Simpson, K.W., Inohara, N., Mobley, H.L.T., Kao, J.Y., Fukuda, S., Barnich, N., Kamada, N., (2020) Dietary l-serine confers a competitive fitness advantage to Enterobacteriaceae in the inflamed gut. Nature Microbiology 5, 116-125. 
Koppolu, V., Vasigala, V.K., (2016) Role of Escherichia coli in Biofuel Production. Microbiol Insights 9, 29-35.

Kustu, S., Hirschman, J., Burton, D., Jelesko, J., Meeks, J.C., (1984) Covalent modification of bacterial glutamine synthetase: physiological significance. Mol Gen Genet 197, 309-317.

Liu, M., Feng, X., Ding, Y., Zhao, G., Liu, H., Xian, M., (2015) Metabolic engineering of Escherichia coli to improve recombinant protein production. Appl Microbiol Biotechnol 99, 10367-10377.

Matthews, R.G., Neidhardt, F.C., (1989) Elevated serine catabolism is associated with the heat shock response in Escherichia coli. J Bacteriol 171, 2619-2625.

McKitrick, J.C., Pizer, L.I., (1980) Regulation of phosphoglycerate dehydrogenase levels and effect on serine synthesis in Escherichia coli K-12. J Bacteriol 141, 235-245.

Pascuan, C., Fox, A.R., Soto, G., Ayub, N.D., (2015) Exploring the Ancestral Mechanisms of Regulation of Horizontally Acquired Nitrogenases. J Mol Evol 81, 84-89.

Perez Di Giorgio, J., Soto, G., Alleva, K., Jozefkowicz, C., Amodeo, G., Muschietti, J.P., Ayub, N.D., (2014) Prediction of aquaporin function by integrating evolutionary and functional analyses. J Membr Biol 247, 107-125.

Peters-Wendisch, P., Netzer, R., Eggeling, L., Sahm, H., (2002) 3-Phosphoglycerate dehydrogenase from Corynebacterium glutamicum: the $\mathrm{C}$-terminal domain is not essential for activity but is required for inhibition by L-serine. Applied Microbiology and Biotechnology 60, 437-441.

Pizer, L.I., Potochny, M.L., (1964) NUTRITIONAL AND REGULATORY ASPECTS OF SERINE METABOLISM IN ESCHERICHIA COLI. J Bacteriol 88, 611-619.

Reitzer, L., (2003) Nitrogen assimilation and global regulation in Escherichia coli. Annu RevMicrobiol $57,155-176$.

Ruprich-Robert, G., Zickler, D., Berteaux-Lecellier, V., Velot, C., Picard, M., (2002) Lack of

mitochondrial citrate synthase discloses a new meiotic checkpoint in a strict aerobe. EMBO J 21, 6440-6451.

Setten, L., Soto, G., Mozzicafreddo, M., Fox, A.R., Lisi, C., Cuccioloni, M., Angeletti, M., Pagano, E., Diaz-Paleo, A., Ayub, N.D., (2013) Engineering Pseudomonas protegens Pf-5 for nitrogen fixation and its application to improve plant growth under nitrogen-deficient conditions. PLoS One 8, e63666. Silva-Rocha, R., Martinez-Garcia, E., Calles, B., Chavarria, M., Arce-Rodriguez, A., de Las Heras, A., Paez-Espino, A.D., Durante-Rodriguez, G., Kim, J., Nikel, P.I., Platero, R., de Lorenzo, V., (2013) The Standard European Vector Architecture (SEVA): a coherent platform for the analysis and deployment of complex prokaryotic phenotypes. Nucleic Acids Res 41, D666-675.

Soto, G., Setten, L., Lisi, C., Maurelis, C., Mozzicafreddo, M., Cuccioloni, M., Angeletti, M., Ayub, N.D., (2012) Hydroxybutyrate prevents protein aggregation in the halotolerant bacterium Pseudomonas sp. CT13 under abiotic stress. Extremophiles 16, 455-462.

Stecher, G., Tamura, K., Kumar, S., (2020) Molecular Evolutionary Genetics Analysis (MEGA) for macOS. Mol Biol Evol 37, 1237-1239.

Stritzler, M., Soto, G., Ayub, N., (2018) Plant Growth-Promoting Genes can Switch to be Virulence Factors via Horizontal Gene Transfer. Microb Ecol 76, 579-583.

Thomas, G., Coutts, G., Merrick, M., (2000) The gInKamtB operon. A conserved gene pair in prokaryotes. Trends Genet 16, 11-14.

van Heeswijk, W.C., Westerhoff, H.V., Boogerd, F.C., (2013) Nitrogen assimilation in Escherichia coli: putting molecular data into a systems perspective. Microbiol Mol Biol Rev 77, 628-695.

Viollier, P.H., Minas, W., Dale, G.E., Folcher, M., Thompson, C.J., (2001) Role of acid metabolism in Streptomyces coelicolor morphological differentiation and antibiotic biosynthesis. J Bacteriol 183, 3184-3192.

Vo,J., Inwood, W., Hayes, J.M., Kustu, S., (2013) Mechanism for nitrogen isotope fractionation during ámmonium assimilation by Escherichia coli K12. Proc Natl Acad Sci U S A 110, 8696-8701.

Vornhagen, J., Sun, Y., Breen, P., Forsyth, V., Zhao, L., Mobley, H.L.T., Bachman, M.A., (2019) The Klebsiella pneumoniae citrate synthase gene, gltA, influences site specific fitness during infection. PLoS Pathog 15, e1008010. 
Yang, D., Park, S.Y., Park, Y.S., Eun, H., Lee, S.Y., (2020) Metabolic Engineering of Escherichia coli for Natural Product Biosynthesis. Trends Biotechnol.

Zalis, E.A., Nuxoll, A.S., Manuse, S., Clair, G., Radlinski, L.C., Conlon, B.P., Adkins, J., Lewis, K., (2019) Stochastic Variation in Expression of the Tricarboxylic Acid Cycle Produces Persister Cells. mBio 10. Zhang, W., Zhang, M., Gao, C., Zhang, Y., Ge, Y., Guo, S., Guo, X., Zhou, Z., Liu, Q., Zhang, Y., Ma, C., Tao, F., Xu, P., (2017) Coupling between d-3-phosphoglycerate dehydrogenase and d-2-

hydroxyglutarate dehydrogenase drives bacterial I-serine synthesis. Proc Natl Acad Sci U S A 114, E7574-E7582.

Zhao, G., Winkler, M.E., (1996) A novel alpha-ketoglutarate reductase activity of the serA-encoded 3phosphoglycerate dehydrogenase of Escherichia coli K-12 and its possible implications for human 2hydroxyglutaric aciduria. J Bacteriol 178, 232-239.

Zhu, M., Dai, X., (2018) On the intrinsic constraint of bacterial growth rate: M. tuberculosis's view of the protein translation capacity. Crit Rev Microbiol 44, 455-464. 


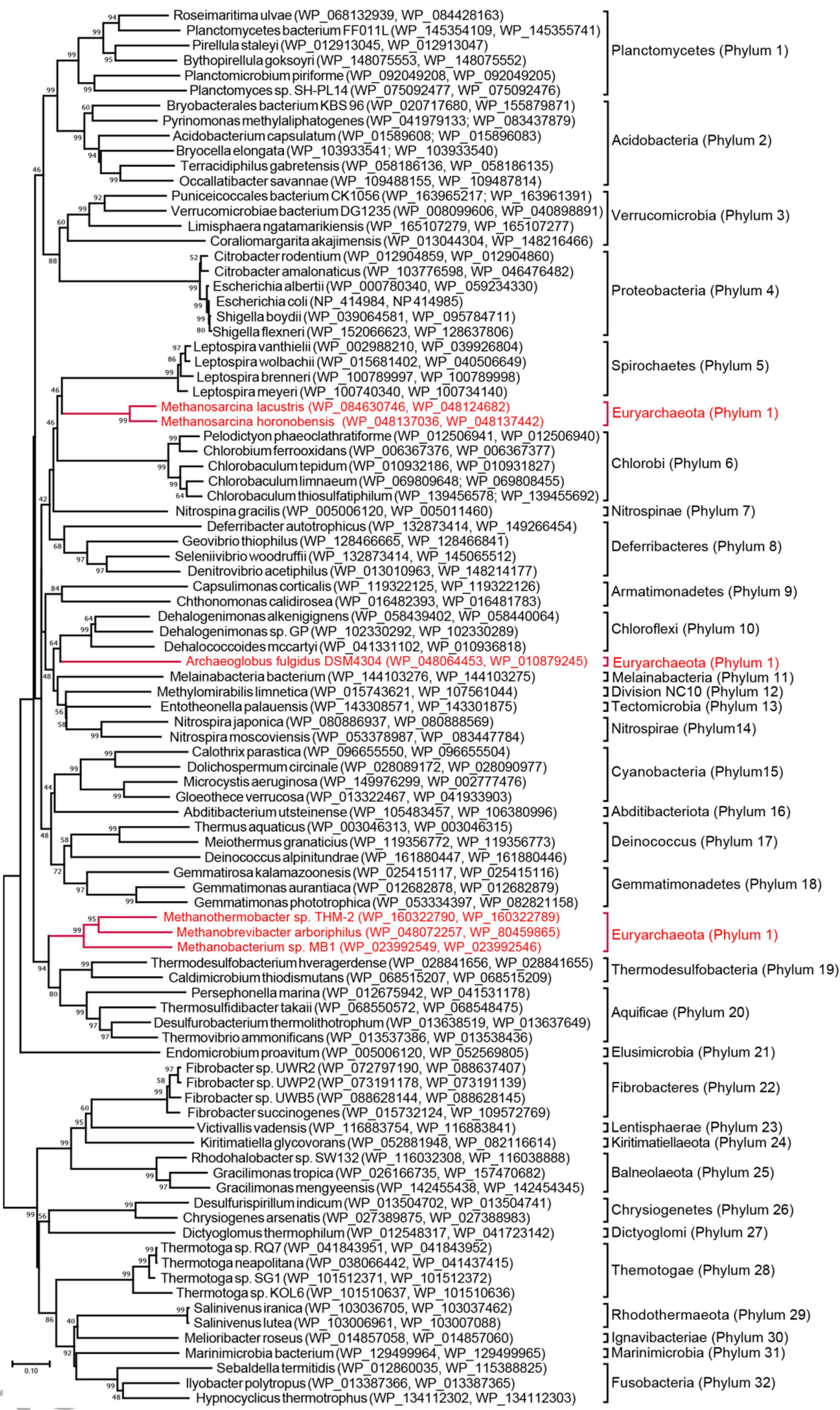

Figure 1. The evolution of the $g / n K$ and amtB genes in prokaryotes. Phylogenetic analysis comparing the consensus tree of the GlnK and AmtB proteins (on the left) and 
bacterial evolution at phylum level (on the right). GlnK and AmtB proteins from Stenosarchaea (M. lacustris and M. horonobensis), Archaeoglobi (A. fulgidus DSM 4304) and Methanomada (Methanothermobacter sp. THM-2, M. arboriphilus and Methanobacterium sp. MB1) subgroups of the archaeal phylum Euryarchaeota (at left) clustered with three different bacterial lineages (on the right). Bootstrap percentages of $40 \%$ or more are indicated at the branch points. 


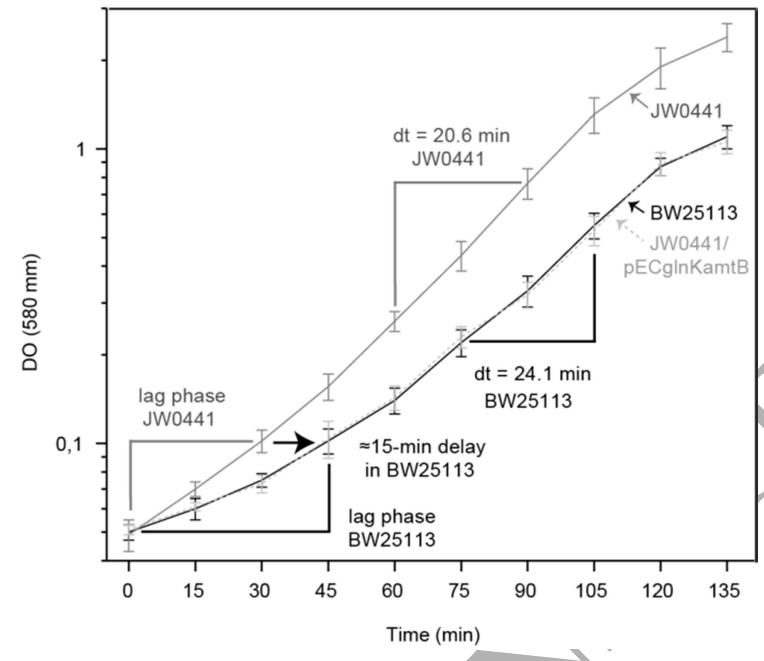

Figure 2. Effects of the mutation of gInKamtB on lag and growth phases of Escherichia coli. The duration of the lag phase and growth rates of Escherichia coli BW25113, its glnKamtB mutant derivative, strain $E$. coli JW0441, and the complemented strain JW0441/pECglnKamtB were evaluated in nutrient-rich LB medium. Values represent mean \pm SD $(n=7)$. Doubling time (dt) was calculated in exponentially growing cells (OD $580 \mathrm{~nm}=0.23-$ 0.76 ). 
(a)

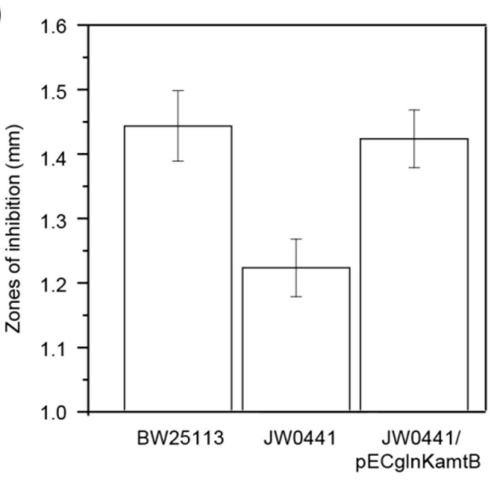

(b)

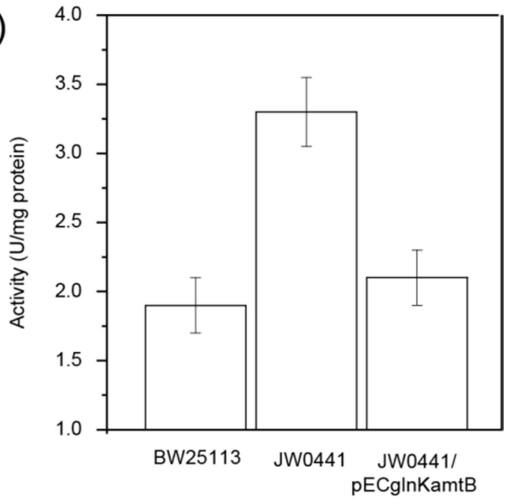

Figure 3. Effect of the gInKamtB mutation on the oxidative stress resistance and the citrate synthase activity of Escherichia coli. (a) The tolerance to $\mathrm{H}_{2} \mathrm{O}_{2}$ of the wild-type strain Escherichia coli BW25113, its gInKamtB mutant derivative, strain E. coli JW0441, and the complemented strain E. coli JW0441/pECgInKamtB growing in nutrient-rich LB medium was evaluated by the disk inhibition assay. Inhibition zones were measured in millimeters. Values represent the mean $\pm S D(n=5)$. (b) The citrate synthase activity of Escherichia coli BW25113, its glnKamtB mutant derivative, strain E. coli JW0441, and the complemented strain JW0441/pECgInKamtB was analyzed in nutrient-rich LB medium in exponentially growing cells (OD $580 \mathrm{~nm}=0.45-0.55)$. Values represent mean $\pm \mathrm{SD}(\mathrm{n}=4)$. 
(a)

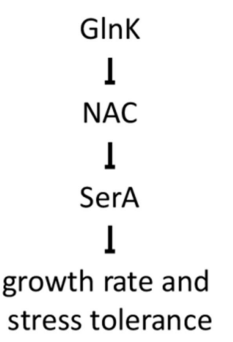

(c)

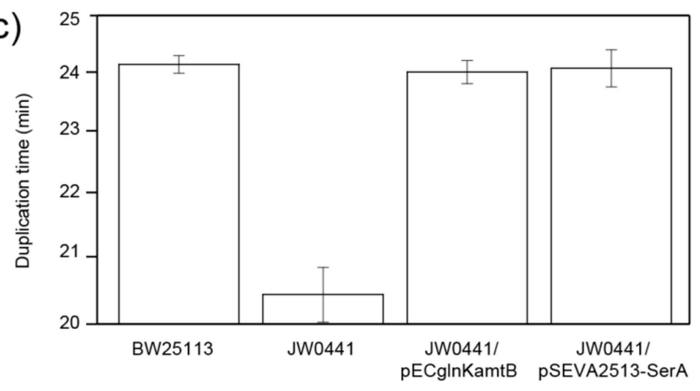

(b)

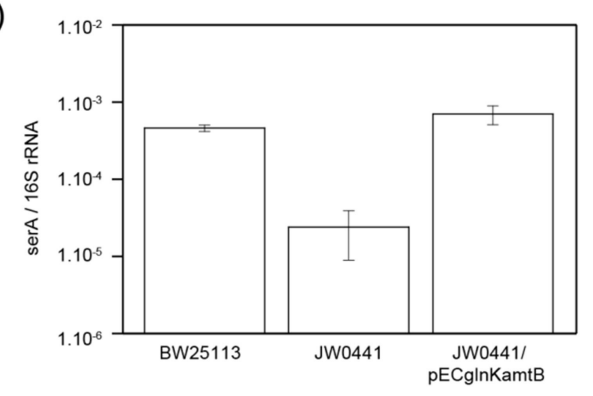

(d)

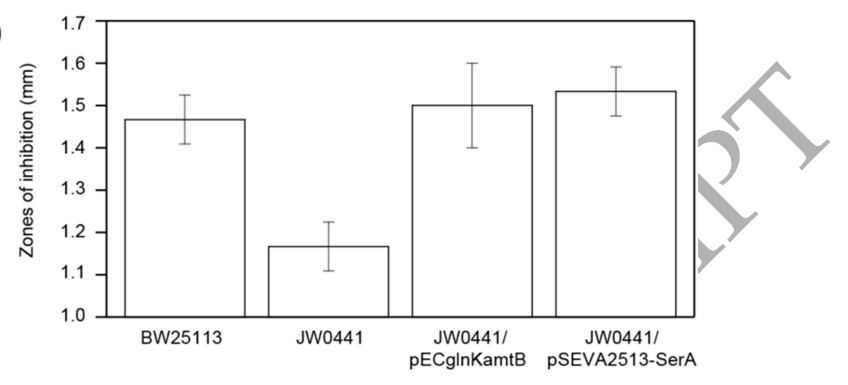

Figure 4. Effects of the mutation of gInKamtB on the expression of the serA gene and the analysis of the relevance of serA expression in bacterial fitness under nutrient-rich conditions. (a) Schematic representation of the hypothetical influence of GInK on the growth rate and stress tolerance via the indirect control of the expression of serA, (b) Real-time RTPCR studies of serA expression in Escherichia coli BW25113, its glnKamtB mutant derivative, E. coli JW0441, and the complemented strain JW0441/pECgInKamtB, (c) growth rates of strains BW25113, JW0441, JW0441/pECgInKamtB and JW0441 transformed with the pSEVA2513-SerA plasmid, analyzed in nutrient-rich LB medium in exponentially growing cells, and (d) tolerance of these strains to $\mathrm{H}_{2} \mathrm{O}_{2}$, studied by the disk inhibition assay. Values represent mean $\pm S D(n=3)$. 
Table 1. Benefits of the presence of the gInKamtB operon in nitrogen-limiting conditions. The growth of the wild type strain Escherichia coli BW25112 and its derived glnKamtB mutant strain Escherichia coli JW0441 was analyzed in minimal solid medium M9 supplemented with different concentrations of ammonium $\left(\mathrm{NH}_{4} \mathrm{Cl}\right)$ under acid $(\mathrm{pH}=5.5)$ or nearly neutral $(\mathrm{pH}=$ 7.4) conditions. Absence of growth (-). Presence of vigorous (++) and moderate $(+)$ growth was defined based on relative colony size.

\begin{tabular}{|c|c|c|c|c|}
\hline \multirow{2}{*}{$\begin{array}{c}\text { NH4Cl } \\
(\mathrm{mM})\end{array}$} & \multicolumn{2}{|c|}{$\begin{array}{c}\text { E. coli } \\
\text { BW25112 }\end{array}$} & \multicolumn{2}{c|}{ E. coli JW0441 } \\
\cline { 2 - 5 } & $\mathrm{pH} \mathrm{5.5}$ & $\mathrm{pH} 7.4$ & $\mathrm{pH} \mathrm{5.5}$ & $\mathrm{pH} \mathrm{7.4}$ \\
\hline 20 & ++ & ++ & ++ & ++ \\
\hline 5 & ++ & ++ & ++ & ++ \\
\hline 1 & ++ & ++ & ++ & ++ \\
\hline 0.5 & ++ & ++ & ++ & ++ \\
\hline 0.25 & ++ & ++ & + & ++ \\
\hline 0.1 & + & ++ & $(-)$ & ++ \\
\hline 0.01 & + & ++ & $(-)$ & ++ \\
\hline
\end{tabular}


Table 2. Importance of the presence of a high-affinity ammonium channel in bacterial growth under nitrogen-limiting conditions. Analysis of growth patterns of Escherichia coli BW25113 and its glnKamtB mutant derivative, strain E. coli JW0441, under extreme nitrogen deficiency (solid M9 minimal medium supplemented with $0.1 \mathrm{mM} \mathrm{NH}_{4} \mathrm{Cl}$ at $\mathrm{pH}=5.5$, selected previously in Table 1 and visualized in Figure S2). E. coli strains JW0441/pECgInKamtB and JW0441/pNOD26 were used for homologous and heterologous complementation assays, respectively. As a control of the presence of bacterial cells in each sample, same aliquots were also plated onto LB agar. Values represent mean \pm SD $(n=3)$.

\begin{tabular}{|c|c|c|c|}
\hline \multirow{2}{*}{ Strains } & \multicolumn{2}{|c|}{$\begin{array}{c}\text { Bacterial growth } \\
\text { (colony-forming units) }\end{array}$} & $\begin{array}{c}\text { Viable cells under } \\
\text { extreme nitrogen } \\
\text { deficiency (M9 / LB) }\end{array}$ \\
\cline { 2 - 4 } & M9 & LB agar (control) & $29 \%$ \\
\hline BW25112 & $42.6 \pm 3.2$ & $145.0 \pm 11.7$ & $0 \%$ \\
\hline JW0441 & Not detected & $129.6 \pm 6.5$ & $24 \%$ \\
\hline JW0441/pECgInKamtB & $30.6 \pm 3.5$ & $128.3 \pm 16.1$ & $92 \%$ \\
\hline
\end{tabular}

